\title{
Artroscopía de rodilla pediátrica. Tres años de experiencia en un Hospital especializado en México
}

\author{
Pediatric knee arthroscopy. Three-year experience at a high specialty hospital in Mexico \\ García-Galicia A,* Jiménez-Reyes M, ${ }^{\ddagger} \S$ Villeda-Gómez SA, ${ }^{\S}$ \\ Hernández-Delgado J,§ Montiel-Jarquín ÁJ,* Martínez-Asención JP§
}

UMAE. Hospital de Traumatología y Ortopedia de Puebla, Centro Médico Nacional «Gral. de Div. Manuel Ávila Camacho» del IMSS.

RESUMEN. Introducción: El diagnóstico y manejo de numerosos trastornos articulares congénitos y traumáticos revisten un problema adicional en la población joven. La artroscopía ha mejorado el tratamiento de estas lesiones cada vez más frecuentes. Objetivo: Describir la experiencia de la aproximación artroscópica en enfermos pediátricos quienes presentan patología de rodilla, admitidos en un tercer nivel de atención en Puebla, México. Material y métodos: Estudio descriptivo, retrospectivo, transversal, realizado en pacientes niños con problemas de rodilla, atendidos por artroscopía, del primero de Marzo 2015 al 28 de Febrero 2018. Se les aplicaron las escalas funcionales de Lysholm e IKDC y de satisfacción funcional de Tegner al momento de la lesión, a los seis y a los doce meses. Se utilizó t de Student y Wilcoxon. Resultados: Se reclutaron 29 jóvenes de cinco a 17 años de edad con mayor porcentaje de mujeres (62.06 \%). El miembro más afectado fue el izquierdo con 55.17\%. Las patologías fueron: menisco discoide, traumatismo de ligamento cruzado anterior, sinovitis inflamatoria idiopática, tumoración, lesión meniscal, daño condral, hiperlaxitud rotuliana. En dichas escalas Lysholm e IKDC se halló mejoría desde el primer semestre. En la escala Tegner hubo incorpo-
ABSTRACT. Introduction: Diagnosis and management of congenital and traumatic articular ailments carry an additional problem in young people. Arthroscopy has improved treatment of these injuries, which are more frequent. Objective: To describe the experience of arthroscopic handling in pediatric patients affected with knee disease, managed at a third level hospital in Puebla, Mexico. Material and methods: Descriptive, retrospective, cross sectional study performed in patients having knee disease, admitted at Unidad Médica de Alta Especialidad Hospital de Traumatología y Ortopedia Puebla, from March first, 2015 to February 28th, 2018. Lysholm and IKDC functional scales and Tegner functional satisfaction scale were applied at zero, six and twelve months. Student's t and Wilcoxon tests were used. Results: 29 young ones of five to 17 years of age became recruited, slightly more women (62.06\%). The most frequently affected limb was the left one, with $55.17 \%$. The disorders found are: discoid meniscus, harm of the anterior cruciate ligament, idiopathic inflammatory synovitis, tumor, meniscal injury, chondral damage, patellar hyperlaxity. Lysholm and IKDC registered improvement from the first semester. Tegner registered their ability to

\section{Nivel de evidencia: IV}

* Unidad Médica de Alta Especialidad Hospital de Especialidades de Puebla, Centro Médico Nacional «Gral. de Div. Manuel Ávila Camacho» del Instituto Mexicano del Seguro Social.

${ }^{\ddagger}$ Facultad de Medicina de la Universidad Popular Autónoma del Estado de Puebla.

$\S$ Unidad Médica de Alta Especialidad Hospital de Traumatología y Ortopedia de Puebla, Centro Médico Nacional «Gral. de Div. Manuel Ávila Camacho» del Instituto Mexicano del Seguro Social.

Correspondencia:

Álvaro José Montiel-Jarquín

Calle 2 norte Núm. 2004, Col. Centro. CP 72000. Puebla, Pue.

Tel: (222) 242-4520, ext. 61315.

E-mail: dralmoja@hotmail.com

Recibido: 10-09-2020. Aceptado: 15-01-2021.

Citar como: García-Galicia A, Jiménez-Reyes M, Villeda-Gómez SA, Hernández-Delgado J, Montiel-Jarquín ÁJ, Martínez-Asención JP. Artroscopía de rodilla pediátrica. Tres años de experiencia en un Hospital especializado en México. Acta Ortop Mex. 2021; 35(1): 28-32. https://dx.doi.org/10.35366/100927

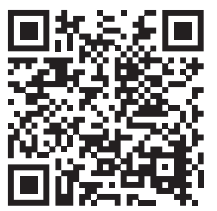


ración completa a sus actividades previas al padecimiento. Se encontraron diferencias estadísticamente significativas $(\mathrm{p} \leq 0.01)$ en las comparaciones de sus funciones a los cero, seis y doce meses de evolución. Conclusiones: Se encontraron daños de frecuencia congénita y traumática, éstas relacionadas al deporte; a través del tratamiento artroscópico hubo resultados funcionales favorables en esa población pediátrica.

Palabras clave: Artroscopía, enfermedades articulares, pacientes pediátricos, padecimientos de rodilla.

\section{Introducción}

La cirugía artroscópica significa grandes beneficios para millones de dolientes en todo el mundo y posiblemente es, desde el siglo pasado, ${ }^{1,2,3}$ una de las más importantes contribuciones de la traumatología a la sociedad.

El diagnóstico y manejo de los trastornos articulares congénitos y traumáticos revisten un problema adicional en la población joven. Existe poca información al respecto, además del concepto erróneo de la elasticidad protectora ante lesiones o de la capacidad persistente de resistencia innata a esos traumatismos en los jóvenes. Esto origina que se valore erróneamente la magnitud del daño, por parte de los mismos pacientes, sus padres y médicos, omitiendo la sospecha de desórdenes articulares que frecuentemente se asocian a trauma repetitivo. Por otro lado, el crecimiento de la artroscopía en la última década y la mejoría del sistema deportivo en México han ocasionado un aumento en la frecuencia de este tipo de trastornos y un abordaje más adecuado. 3,4

Los padres o tutores de tales pacientes -niños o adolescentes- consideran que la cirugía artroscópica posee el mismo nivel de riesgos que las cirugías abiertas, en las cuales se requiere del uso de anestesia general y tiene los mismos peligros inherentes que cualquier otro procedimiento quirúrgico ortopédico. Sin embargo, en dichos infantes aquejados, la eficacia diagnóstico-terapéutica de la técnica artroscópica ya ha sido ampliamente demostrada. ${ }^{5}$

Por otra parte, los requerimientos técnicos y de equipo necesarios para estos procedimientos artroscópicos y la falta de personal capacitado y con experiencia en el método artroscópico, ocasiona que la información documentada de la casuística sea más bien escasa.

En esta investigación se aborda la tarea de valorar el tipo de lesión y su correlación clínico-artroscópica en pacientes menores de 18 años sometidos a intervención artroscópica de rodilla, revelando la eficacia para un tratamiento oportuno además de correctivo. Se tiene como objetivo describir esta experiencia utilizando el manejo artroscópico de enfermos de corta edad con padecimientos de dicha articulación, admitidos en un tercer nivel de atención. go back to previous daily occupations. On the Lysholm scale, there was improvement at six months. On the Tegner scale there was complete incorporation to their activities (previous to the presentation and surgical intervention). At six months subsequent to treatment, the IKDC evidenced improvement and recovery, and reintegration to their activities. Statistically significant differences $(p \leq 0.01)$ resulted, in measurements at zero, six and 12 months. Conclusions: Congenital and traumatic illnesses were found, the latter ones sports related. Arthroscopic approach registered positive functional results in these children.

Keywords: Arthroscopy, articular diseases, pediatric patients, knee disease.

\section{Material y métodos}

El presente trabajo fue aprobado por el Comité Local de Investigación y Ética en Investigación en Salud No. 2102 del IMSS, con número de registro R-2017-2105-28. Se realizó un estudio descriptivo, transversal, unicéntrico en pacientes pediátricos con patología de rodilla operados por artroscopía del primero de Marzo 2015 al 28 de Febrero 2018. Se les aplicaron las escalas funcionales de Lysholm e IKDC y de satisfacción funcional de Tegner al momento de la lesión, y luego a los seis y 12 meses.

Se incluyeron jóvenes quienes sufrían avería intraarticular congénita de tal articulación, traumática y de origen a determinar con contraindicación de intervención quirúrgica abierta.

Se procesaron los datos mediante estadística descriptiva y análisis de varianza utilizando ANOVA, Kruskal-Wallis. Los procesamientos estadísticos se realizaron en el programa Sigmastat.

\section{Resultados}

Se estudiaron 29 pacientes de cinco a 17 años de edad. El miembro pélvico mayormente afectado fue el izquierdo (Tabla 1). En esta exploración, las patologías fueron: menisco discoide, lesión de ligamento cruzado anterior (LCA), sinovitis inflamatoria idiopática (corroborada por análisis anatomopatológico), fractura de espina tibial (postraumática), tumoración, daños meniscales, lesiones condrales e hiperlaxitud rotuliana (Figura 1).

Al aplicar la métrica de Lysholm se observó la mejoría de esos enfermos en la realización de sus ocupaciones diarias y deportivas evidenciando una rehabilitación desde el semestre inicial (Figura 2).

$\mathrm{Al}$ emplear la encuesta de Tegner se percibió la incorporación a sus asuntos anteriores a la lesión y recuperación completa en sus funciones.

En la valoración del formulario del Comité Internacional de Documentación sobre la Rodilla (por sus siglas en inglés IKDC) se demuestra mejoría y recuperación posterior 
al tratamiento incluso en el primer semestre, siendo buena y excelente, alcanzando integración total a sus labores, incluyendo actividades previas (Figura 3).

Las diferencias encontradas en la aplicación de las escalas funcionales a los cero, seis y doce meses, así como su significancia se muestran en la Tabla 2. Destaca que esas comparaciones registraron gran significado estadístico, salvo en la comparación entre cero y seis meses en la puntuación de Lysholm como variable cualitativa, utilizando la prueba de Wilcoxon. Sin embargo, la misma escala con valores cuantitativos y la escala IKDC encontraron alta significancia en todas las comparaciones (Tabla 2).

\section{Discusión}

Actualmente, la artroscopía juega un papel definitivo en el diagnóstico y atención de los traumatismos de rodilla en niños y adolescentes. Las disfunciones más frecuentes de esta unión, halladas por técnica artroscópica a estas edades, corresponden a traumatismos condrales y osteocondrales (45\% en menores y $29 \%$ en adolescentes), seguidas de deterioros del LCA y meniscales, incrementándose acorde a la edad. La aproximación diagnóstica a dichos problemas en esa articulación mediante la inspección clínica es de aproximadamente 25-56\%, mientras que con el método artroscópico es hasta $99 \% .{ }^{6}$ Esto concuerda con la casuística de la presente exposición, revelando mayor frecuencia en edades de 13 a 17 años y en lesiones de sinovitis, daños en los meniscos y de LCA.

Existen reportes de intensificación gradual en la incidencia de estas dolencias en mujeres, concomitante con el aumento de participación de ellas en los deportes; sin embargo, en población hispana, se sigue registrando mayormente la prevalencia de daños de rodilla en varones, entre 62 y 71\%. ${ }^{7}$ Curiosamente, en el presente estudio las niñas evidenciaron una frecuencia elevada (62\%), quizá debido a cuestiones del régimen de atención del hospital, semicerrado limitado a trabajadores y sus familiares y a una muestra pequeña.

\begin{tabular}{|c|c|}
\hline \begin{tabular}{|} 
Tabla 1: Características de los pacientes \\
pediátricos con patología de rodilla.
\end{tabular} \\
\hline Edad (años) & $\mathrm{n}(\%)$ \\
\hline $5-6$ & $2(8)$ \\
$9-12$ & $9(30)$ \\
\hline $13-17$ & $18(62)$ \\
\hline Género & $\mathrm{n}(\%)$ \\
\hline Masculino & $11(38)$ \\
\hline Femenino & $18(62)$ \\
\hline Miembro pélvico afectado & $(\%)$ \\
\hline Derecho & 44.82 \\
\hline Izquierdo & 55.17 \\
\hline
\end{tabular}

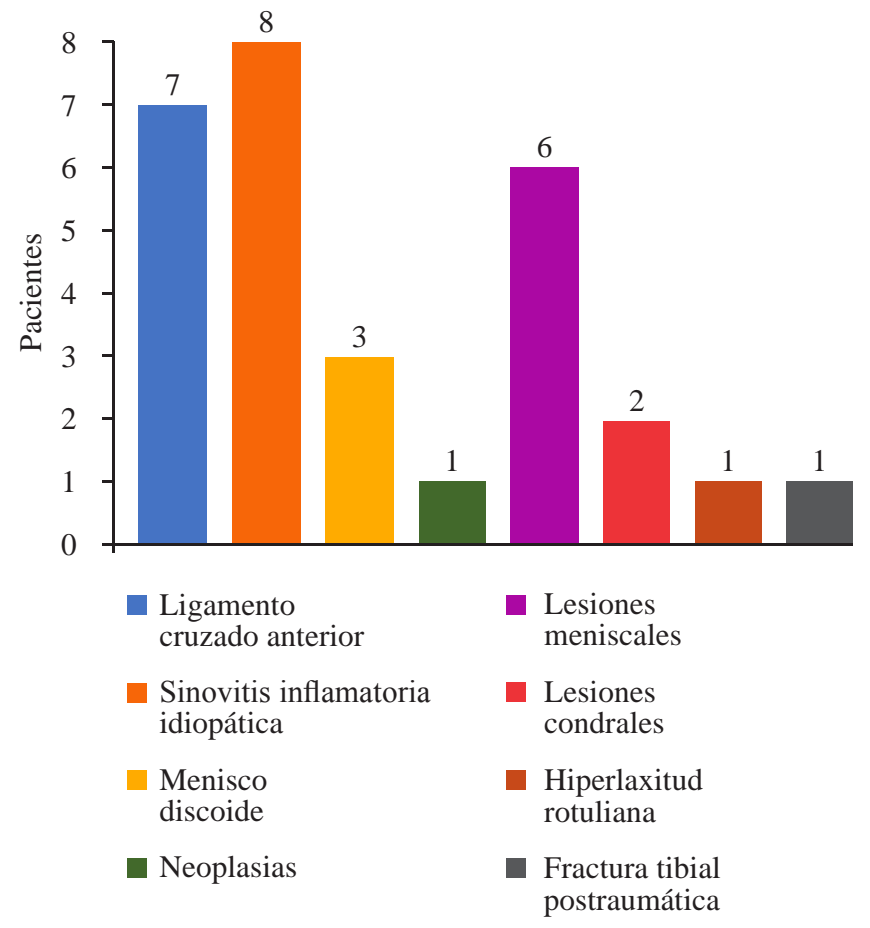

Figura 1: Patologías de rodilla en pacientes pediátricos.

La presencia de hemartrosis en las contusiones de tal coyuntura puede asociarse a lesiones de mayor complicación, especialmente de ligamento cruzado anterior, destacando la etiología deportiva. Ello justificaría la recomendación del procedimiento artroscópico. ${ }^{8}$ El incremento de incidencia de traumatismos deportivos en niños se debe probablemente al deseo de los padres de que sus hijos practiquen deporte en edades tempranas. En cuanto a lesiones ligamentosas, poco frecuentes en esta serie de pacientes, pueden encontrarse aisladas o asociadas a deterioros fisarios o fracturas de huesos largos. ${ }^{8}$ En este caso, se tuvo un evento de inestabilidad multiligamentaria. Éste fue en una paciente escolar donde el mecanismo del trauma fue el apilamiento de sus compañeros sobre ella mientras jugaban. Requirió dos tiempos quirúrgicos artroscópicos persiguiendo la estabilización de la rodilla afectada.

La artroscopía ha evidenciado ser muy eficaz, es el método de elección en tratamiento quirúrgico de las lesiones meniscales. En jóvenes, permite la reparación de daños del menisco discoideo por su meniscectomía parcial y la realización de sinovectomía parcial. En aquellos casos de osteocondritis disecante facilita la extracción de cuerpos libres. Permite curación total en la mayoría de esos casos tratados de reparación o reconstrucción por artroscopía de las alteraciones de LCA o bien de avulsiones osteoligamentarias, logrando restaurar la estabilidad de la articulación. No parece ser tan efectiva la aproximación artroscópica en el manejo del dolor femoropatelar. ${ }^{9}$ En este artículo, los detrimentos en los meniscos y de menisco discoide, que conjuntamente hacen $34.5 \%$, registraron gran mejoría con la atención artroscópica de acuerdo con las escalas funcionales. Es notoria la alta prevalencia de daños asociados en dichos meniscos, 
quizá debido a la larga lista de espera en este servicio hospitalario (siendo la artroscopía un método de alta demanda y el personal capacitado en la técnica artroscópica de rodilla aún limitado), siguiendo lo encontrado por Gallagher de aumento en deterioros meniscales en pacientes con dilación en el procedimiento. ${ }^{10}$

En los eventos de patología infecciosa e inflamatoria de rodilla, la intervención artroscópica es útil a fin de realizar un diagnóstico diferencial ya que permite la evaluación completa. Por ejemplo, en la artritis reumatoide juvenil facilita obtener información relevante mucho antes que por radiología, visualizando la lesión del cartílago articular y la severidad de la inflamación sinovial. Además posibilita, si se precisa, efectuar una sinovectomía en el mismo acto artroscópico. ${ }^{11}$ En el presente reporte, la artroscopía originó la mejoría de funciones en los ocho casos (27.5\%) de sinovitis inflamatoria idiopática. Se tuvo especial cuidado en torno a la cantidad de solución que se irrigaba buscando evitar la distensión capsular.
Una característica de la población estudiada fue la alta prevalencia de lesiones meniscales asociadas, del orden de $27.58 \%$, pudiendo ser por la existencia de las listas de espera quirúrgicas y a la tardanza con la cual acuden dichos pacientes. Son muy numerosas las publicaciones que abordan este tema y que revelan que estos traumatismos meniscoligamentosos son frecuentes con una rodilla inestable. La presencia de estas lesiones en el momento de la cirugía puede repercutir de forma negativa sobre los resultados funcionales.

En general, el método artroscópico tiene la ventaja de su baja morbilidad, mínimas cicatrices y una precoz recuperación de la funcionalidad. ${ }^{12}$ La presente serie no arrojó consecuencias adversas ni complicaciones asociadas al evento artroscópico.

En esta unidad médica, en pacientes de poca edad, se encontraron primordialmente disfunciones de etiología congénita y traumática. Estas últimas ligadas al deporte; la manipulación de las mismas a través de abordaje artroscó-

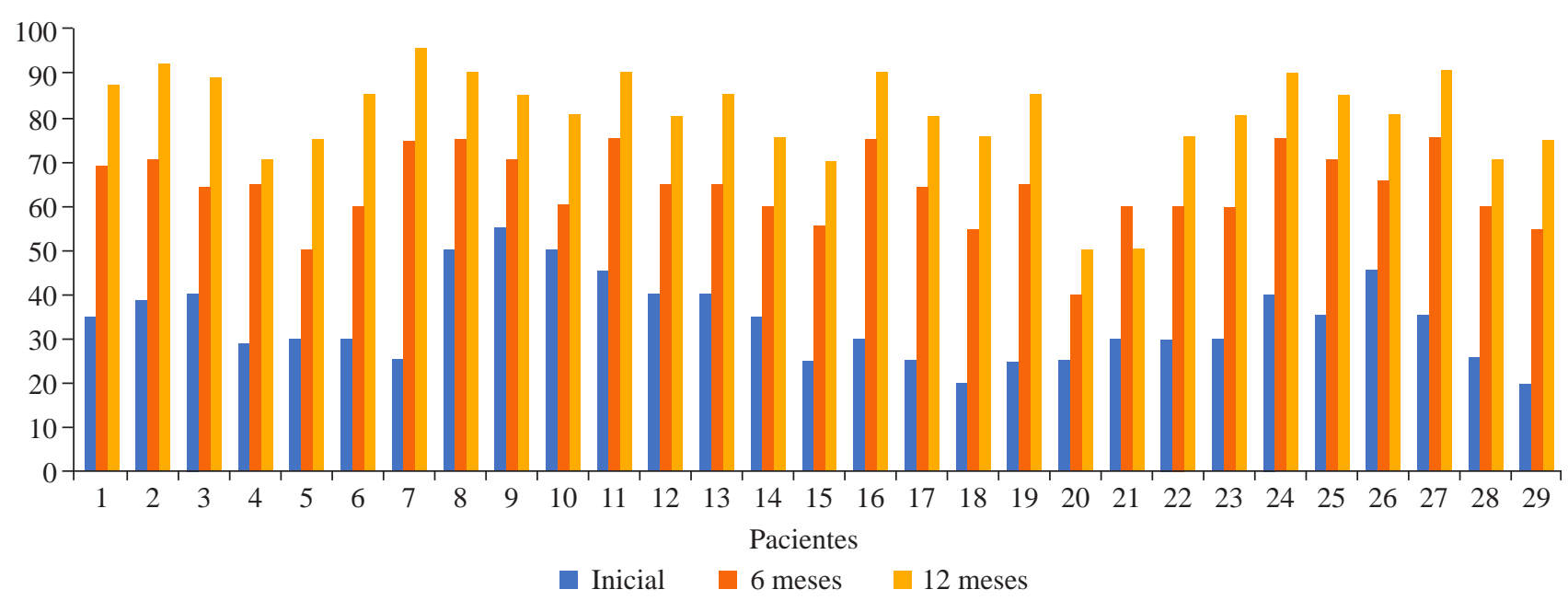

Figura 2: Puntajes de cada paciente en la escala de Lysholm al inicio, 6 y 12 meses.

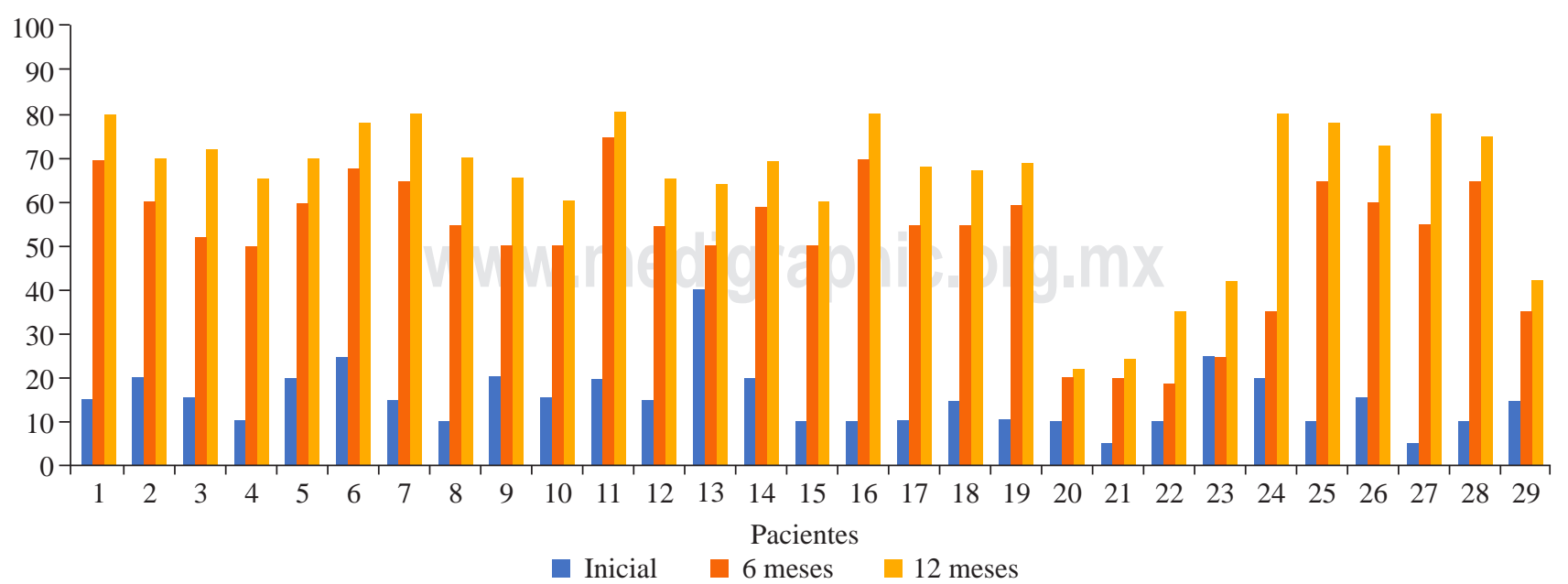

Figura 3: Puntajes de cada paciente en la escala IKDC al inicio, 6 y 12 meses. 
Tabla 2: Resultados estadísticos en escalas de Lysholm e International Knee Documentation Committee aplicadas a los 0, 6 y 12 meses.

\begin{tabular}{|c|c|c|c|c|c|}
\hline Tiempo de Aplicación (meses) & Media de diferencias & Prueba de t de Student & $\mathrm{p}$ & Prueba de Wilcoxon Z & $\mathrm{p}$ \\
\hline \multicolumn{6}{|c|}{ Lysholm } \\
\hline 0 y 6 & $-38,138$ & $-14,716$ & 0.00 & -2.000 & 0.46 \\
\hline 0 y 12 & $-49,586$ & $-16,521$ & 0.00 & -4.000 & 0.00 \\
\hline 6 y 12 & $-11,448$ & $-15,061$ & 0.00 & -3.464 & 0.001 \\
\hline \multicolumn{6}{|c|}{ IKDC } \\
\hline 0 у 6 & $-38,138$ & $-14,716$ & 0.00 & & \\
\hline 0 y 12 & $-49,586$ & $-16,521$ & 0.00 & & \\
\hline 6 y 12 & $-11,448$ & $-15,061$ & 0.00 & & \\
\hline
\end{tabular}

pico registró desenlaces funcionales positivos. Es importante considerar no solamente un adecuado tratamiento y una apropiada técnica quirúrgica, sino también la capacidad de regeneración y plasticidad del paciente pediátrico. Además, ello incluye la exploración y valoración extensas de dicho paciente a ser candidato a manejo artroscópico.

\section{Conclusiones}

El uso de la cirugía artroscópica en pacientes pediátricos afectados por lesiones de rodilla es recomendable, ya que produce una excelente recuperación funcional en los niños.

Referencias

1. Zamudio-Carrera JE, Martínez-Molina OA. Correlación clínicaartroscópica en lesiones de rodilla en pacientes pediátricos. Acta Ortop Mex. 2008; 22(3): 180-8.

2. Cotran RS, Kumar V, Collins T. Articulaciones. En: Robbins. Patología estructural y funcional. $8^{\mathrm{a}}$ ed. Madrid: McGraw-Hill Interamericana; 2000.

3. Castro S, Yañez A, Harfush A, Cassis N. Artroscopía de la rodilla en niños y adolescentes. Experiencia de 38 casos. Rev Mex Ortop Traum. 1994; 8(4): 153-5.

4. Hagino T, Ochiai S, Senga S, Tamashita T, Wako M. Ando T, et al. Arthroscopic treatment of symptomatic discoid meniscus in children. Arch Orthop Trauma Surg. 2017; 137: 89-94.
5. Alvarado-Pérez F, Carriwero-Rico EG, Acevedo-Méndez A, Villanueva Sámano M. Menisco discoide lateral. Diagnóstico y tratamiento artroscópicos. Rev Mex Ortop Traum. 1994; 8(5): 214-19.

6. Ayala-Mejías JD, García-Estrada GA, Alcocer-Pérez-España L. Lesiones del ligamento anterior. Acta Ortop Mex. 2014; 28(1): 57-67.

7. Heyworth BE, Kocher MS. The young athlete. In: Miller M, Thompson S. DeLee, Drez and Miller's orthopaedic sports medicine. Fifth ed. Elsevier Inc.; 2019, 1606-15.

8. Damil-Castro R, Cantere-Oceguera D, Lovaina-Barzaga G. Artroscopía de rodilla en niños y adolescentes bajo régimen ambulatorio: 5 años de experiencia. Rev Cubana de Reumatol. 2001; 3(1): 25-9.

9. Crider R. Lesiones del deporte en niños. Rev Cubana Ortop Traumatol. 1996; 10(1): 89-90.

10. Gallacher PD, Gilbert RE, Kanes G, Roberts SNJ, Rees D. Outcome of meniscal repair prior compared with concurrent ACL reconstruction. Knee. 2012; 19(4): 461-3.

11. Schmitt A, Batisse F, Bonnard C. Results with all-inside meniscal suture in pediatrics. Orthop Traumatol Surg Res. 2016; 102(2): 20711.

12. Damil-Castro R, Checo-González A. Diagnóstico clínico por artroscopía de afecciones de la rodilla en niños y adolescentes. Rev Cubana de Reumatol. 2002; 4(1): 37-41.

Financiamiento: Los autores no recibieron ayuda o financiación para el presente trabajo; asimismo, ellos declaran la inexistencia de conflictos de interés. 